\title{
Análisis del IMC y Somatotipo en una Muestra de Adolescentes con Sobrepeso y Obesidad en Temuco-Chile
}

\author{
Analysis of BMI and Somatotype in a Sample of Adolescents \\ were Overweight and Obesity in Temuco-Chile \\ "Héctor Silva M. ; "Erika Collipal L.; *** Cristian Martínez \& **Ivonne Torres
}

\begin{abstract}
SILVA, M. H.; COLLIPAL, L. E.; MARTÍNEZ, C. \& TORRES I. Análisis del IMC, somatotipo en una muestra de adolescentes con sobrepeso y obesidad en Temuco-Chile.Int. J. Morphol., 26(3):707-711, 2008.

RESUMEN: El sobrepeso y la obesidad en Chile se han convertido en una epidemia que sobrepasa todos los sectores sociales. Los factores más importantes han sido el cambio del estilo de vida, económicos, sedentarismo, genéticos, entre otros. El propósito de este estudio fue analizar el índice de msa corporal (IMC) y somatotipo en una muestra de adolescentes con sobrepeso y obesidad de la ciudad de Temuco-Chile. Evaluamos 50 sujetos de ambos sexos (19 hombres 31 mujeres) de 14 a 16 años de edad, de colegios municipalizados de la ciudad de Temuco, IX Región, Chile. Los sujetos fueron evaluados en sus propios colegios. Para obtener el somatotipo utilizamos el método antropométrico de Heath \& Carter, efectuándose la clasificación del IMC de acuerdo a las normas de la OMS. Las mujeres resultaron ser más endomórficas que los hombres, siendo esta diferencia estadísticamente significativa. Los componentes mesomórfico y ectomorfico no presentaron diferencias estadísticamente significativas según el sexo. El IMC mostró un valor de 27 . La muestra presentó un predominio del componente endomesomórfico para hombres y mujeres pero, además, en ambos hay predominio de este componente. Estos resultados demostraron la existencia de una gran cantidad de tejido adiposo en los adolescentes, con el consecuente aumento de peso y obesidad. Esto último, producto de la falta de actividad física, cambios de los hábitos alimenticios y diversos factores adicionales tales como padres obesos y aspectos genéticos.
\end{abstract}

PALABRAS CLAVE: Indice de masa corporal (IMC); Somatotipo.

\section{INTRODUCCIÓN}

La mayoría de las investigaciones indican que la obesidad y el sobrepeso se han convertido en un problema de salud pública en los países industrializados (Andersen, 2000; Ebbeling et al., 2002; Caitlin \& Fergus, 2005, Veugelers \& Fitzgerald, 2005). La obesidad y el sobrepeso se han incrementado en forma alarmante en Chile en las últimas décadas y no sólo en los adultos, sino también, en la población de edad escolar.

De acuerdo a esto, las tasas de prevalencia estiman que en USA el sobrepeso en los adultos es $55,9 \%$ y que el $30,5 \%$ son obesos, de acuerdo al IMC (Flegal et al., 2002). En cuanto a la población de adolescentes, las tasas de obesidad y sobrepeso no son diferentes y van en aumento, ya que según el NHANES III (1988-1994) y el NHANES (19992000 ) indican que el $15,8 \%$ de niños de 6-11 años y el 16,1\% de los adolescentes de edad entre 12-19 están con sobrepeso.
La obesidad constituye en la actualidad la enfermedad nutricional más prevalente en la población $<20$ años. WHO, 2003

En la población escolar chilena, la tendencia secular muestra que la obesidad se ha casi triplicado en varones y mujeres, al comparar estudios realizados en la década del 80 y del 90 (Muzzo et al., 1999).

El IMC es considerado el mejor indicador antropométrico para diagnosticar el sobrepeso y la obesidad en niños entre los 2 y los 18 años. Según Burrows et al. (2004) las diferencias en el IMC por estadio de Tanner observadas, sugieren que en la evaluación individual de las mujeres y varones, con madurez biológica por fuera de los rangos de normalidad (maduradores tempranos y tardíos), debería considerarse el desarrollo puberal alcanzado, para

\footnotetext{
* Dpto. de Ciencias Básicas, Unidad de Anatomía. Universidad de La Frontera, Chile.

** Dpto. de Educación Física Deportes y Recreación. Universidad de La Frontera, Chile.

Proyecto E.P. No 120542. Dirección de Investigación y Desarrollo, Universidad de La Frontera, Temuco, Chile.
} 
una calificación más adecuada del estado nutricional a través del IMC; además, sugiere que los cambios metabólicos ocurridos en esta etapa de la vida demuestran que la sensibilidad insulínica se asocia a la cantidad y distribución de la grasa corporal y a un perfil lipídico de riesgo cardiovascular, desde edades tempranas. Tanto en prepúberes y púberes, la menor sensibilidad insulínica se asoció a un perfil lipídico más aterogénico y a mayores niveles de glicemia, tanto basal como postestímulo. La distribución centrípeta y la cantidad de grasa corporal (33\% en los prepúberes y $37 \%$ en los púberes), serían factores asociados a un mayor riesgo de expresar una resistencia insulínica (Burrows et al., 2005).

En la actualidad, existen varios estándares para IMC en niños y adolescentes realizados en diferentes países y éstos tienen diferencias significativas a igual percentil. Los estándares americanos muestran una mayor ponderosidad para los percentiles 50 y 95 que los nacionales, especialmente en los varones. El criterio actual para el diagnóstico de la obesidad en el niño y adolescente es más estadístico que biológico, lo que obliga a elaborar un estándar de aplicación universal si queremos utilizar la distribución percentilar, o de lo contrario sería necesario identificar para las diferentes edades los valores equivalentes a los IMC de riesgo y obesidad (27,0 y 30,0) del adulto, ya que estos valores están basados en un criterio más biológico que estadístico (White et al., 1995).

El somatotipo ha sido estudiado en poblaciones normales de diferentes edades, sexo y niveles socioeconómicos para conocer la biotipología de estos grupos humanos. (Katzmarzyk et al. 1998, 1999) Valores específicos de sus componentes han sido correlacionados en diferentes patologías tales como, cáncer de mama, cardiopatías, escoliosis y obesidad. (Magnusson et al., 1998)

El análisis de los pliegues cutáneos permite estimar el contenido de masa grasa y masa libre de grasa en los diferentes individuos y, consecuentemente, inferir los requerimientos nutricionales. (Toro \& Almagià, (1989); Depress (1997); Swan \& McConnell (1999).
En Chile, en estudios poblacionales efectuados por Toro et al. (1983) en la ciudad de Valparaíso, encontraron que las mujeres eran más endomórficas que los hombres y que existía una gran variedad de somatotipos en la población.

El propósito de este estudio fue analizar el somatotipo e IMC de una población adolescente con sobrepeso y obesidad de la ciudad de Temuco, IX Regi;on de Chile.

\section{SUJETOS Y MÉTODO}

Evaluamos el somatotipo e IMC en 50 adolescentes (19 hombres y 31 mujeres) con sobrepeso y obesidad, con edades entre 14 y 16 años, alumnos de colegios urbanos municipalizados de la ciudad de Temuco de la IX Región, Chile.

Los sujetos fueron evaluados en sus colegios y los datos registrados en una ficha. Los instrumentos de medición utilizados fueron: banco de antropometría, estadíometro de pared, adipómetro de Harpender, antropómetro de Lafayette y cinta métrica Sany.

Para la obtención del somatotipo utilizamos el método de Heath \& Carter. Para el análisis de los datos, empleamos el programa SPSS 10.0.

\section{RESULTADOS}

Heath \& Carter caracterizaron el somatotipo en los componentes endomórfico, mesomórfico, ectomorfico. Los valores de los componentes del somatotipo y del IMC por edad y sexo se encuentran en las Tablas I a VI.

Tabla I. Valores de centralización de edad, peso y estatura de una muestra de adolescentes de ambos sexos de la ciudad de Temuco, Chile. $\mathrm{n}=50$.

\begin{tabular}{lcccccc}
\hline & $\begin{array}{c}\text { Promedio } \\
\text { hombres }\end{array}$ & D.S. & $\begin{array}{c}\text { Promedio } \\
\text { mujeres }\end{array}$ & D.S. & $\begin{array}{c}\text { Promedio } \\
\text { general }\end{array}$ & D.S. \\
\hline Edad (años) & 14,5 & 0,7 & 14,7 & 0,6 & 14,6 & 0,6 \\
Peso (Kg) & 77,0 & 12,8 & 71,6 & 7,5 & 73,7 & 12,8 \\
Estatura(cm) & 164,0 & 4,7 & 161,0 & 6,2 & 162,0 & 7,7 \\
\hline
\end{tabular}


SILVA, M. H.; COLLIPAL, L. E.; MARTíNEZ, C. \& TORRES I. Análisis del IMC, somatotipo en una muestra de adolescentes con sobrepeso y obesidad en Temuco-Chile. Int. J. Morphol., 26(3):707-711, 2008 .

Tabla II. Clasificación del somatotipo e índice de masa corporal en adolescentes de ambos sexos según edad en Temuco, Chile. n= 50.

\begin{tabular}{lcccccccccc}
\hline $\begin{array}{l}\text { Edad } \\
\text { (años) }\end{array}$ & \multicolumn{2}{c}{$\mathrm{n}$} & \multicolumn{2}{c}{ Endomorfía } & \multicolumn{2}{c}{ Mesomorfía } & \multicolumn{2}{c}{ Ectomorfía } & \multicolumn{2}{c}{ IMC } \\
& Hombres Mujeres & Hombres & Mujeres & Hombres & Mujeres & Hombres & Mujeres & Hombres & Mujeres \\
\hline 14 & 11 & 13 & 7,4 & 8,1 & 3,9 & 4,0 & 0,6 & 0,4 & 27,9 & 26,2 \\
15 & 6 & 14 & 7,3 & 8,1 & 4,0 & 4,6 & 0,6 & 0,4 & 27,8 & 28,1 \\
16 & 2 & 4 & 7,7 & 8,7 & 4,6 & 2,7 & 0,2 & 0,1 & 34,8 & 28,7 \\
\hline
\end{tabular}

Tabla III. Clasificación del somatotipo e índice de masa corporal, en adolescentes de ambos sexos según edad en Temuco, Chile $\mathrm{n}=50$.

\begin{tabular}{lccccc}
\hline $\begin{array}{l}\text { Edad } \\
\text { años }\end{array}$ & $\mathrm{n}$ & Endomorfía & Mesomorfía & Ectomorfía & \multicolumn{1}{c}{ IMC } \\
\hline 14 & & $\mathrm{X}$ & $\mathrm{X}$ & $\mathrm{X}$ & $\mathrm{X}$ \\
15 & 24 & 7,7 & 4 & 0,5 & 27,0 \\
16 & 20 & 7,8 & 4,4 & 1,0 & 28 \\
\end{tabular}

Tabla IV. Componentes del somatotipo en una muestra de adolescentes de ambos sexos de la ciudad de Temuco-Chile. $\mathrm{n}=50$

\begin{tabular}{lcccc}
\hline & \multicolumn{2}{c}{ Hombres } & \multicolumn{2}{c}{ Mujeres } \\
& Promedio & S.D. & Promedio & S.D. \\
\hline Ectomorfía & 0,6 & 0,4 & 0,4 & 0,3 \\
Mesomorfía & 4,0 & 1,2 & 4,1 & 1,1 \\
Endomorfía & 7,4 & 0,8 & 8,1 & 0,6 \\
\hline
\end{tabular}

Tabla V. Distribución del índice de masa corporal de una muestra de adolescentes de ambos sexos de la ciudad de Temuco-Chile. $\mathrm{n}=50$

\begin{tabular}{crrrrrr}
\hline & & Sobrepeso & \multicolumn{2}{c}{ Obeso } & \multicolumn{2}{c}{ Total } \\
\hline & $\mathrm{n}$ & $\%$ & $\mathrm{n}$ & $\%$ & $\mathrm{n}$ & $\%$ \\
\hline Hombres & 4 & 8 & 15 & 30 & 19 & 38 \\
Mujeres & 13 & 26 & 18 & 36 & 31 & 62 \\
\hline
\end{tabular}

La prueba t de student aplicada a la muestra general para las variables IMC y componentes del somatotipo, resultó estadísticamente significativa a favor de las mujeres en la variable endomorfía; en cambio, las variables mesomorfía, ectomorfía e IMC no arrojaron diferencias significativas entre ambos sexos.

\section{DISCUSIÓN}

En Chile, se ha encontrado gran variedad de somatotipos, que se distribuyen en los sectores endomesomórficos y mesoendomórficos. En la muestra se observó un predominio del componente endomesomórfico, lo que indica la existencia de un alto componente de tejido adiposo, concordante con el aumento de peso y con la obesidad de los adolescentes. La literatura señala que las mujeres son más endomórficas en relación a los varones, (Toro et al., 1983; Almagià et al., 1996, 1997; Silva et al., 2003; Swang McConnell, 1999) lo que concuerda con nuestro estudio. Las mujeres presentan diferencias estadísticamente significativas, siendo más endomórficas que los hombres. Wang et al., 1994); Almagià et al., 1996, el componente endomórfico es también predominante en los varones, ya que en general el componente mesomórfico es relevante en los hombres, lo que se debe a que la muestra se encuentra en el rango de obesos y sobrepeso. (Toro et al.; Wang et al.; Almagià et al., 1996 y Swang \& McConnell). 
Los pliegues cutáneos son excelentes indicadores para valorar el somatotipo, el cual da cuenta de los tres componentes para poder determinar el contenido de graso y correlacionar estos valores con el IMC. La muestra presenta valores de endomorfía máximos de acuerdo a la escala de medición tanto para hombres y mujeres. Debido a esto, se puede inferir que ellos tienen un factor más para padecer, en el futuro, enfermedades crónicas no transmisibles, tales como, hipertensión arterial y/o diabetes.

Creemos que un control de la obesidad en los colegios, tiene que ser una prioridad, es necesrio crear conciencia fundamentalmente en los padres, así como también, en profesores, y en todo el ámbito educativo para instaurar normas sobre el control de la obesidad en los colegios, ya que esta enfermedad se instaura en los primeros estadios de la vida. Si la tendencia de obesidad se mantiene y progesa, se podrá en el futuro pesquisar la presentación de complicaciones metabólicas a temprana edad. Por tanto, es oportuno mantener una vigilancia más estrecha de las condiciones de estos adolescentes. Los hábitos alimenticios y el nivel de conocimientos de los alimentos que poseen los padres, han mostrado ser deficientes y la percepción que tienen los padres de la obesidad de sus hijos es una variable también importante, como lo muestran los estudios nacionales.

SILVA, M. H.; COLLIPAL, L. E.; MARTÍNEZ, C. \& TORRES I. Analysis of BMI and somatotype in a sample of adolescents were overweight and obesity in Temuco - Chile.Int. J. Morphol., 26(3):707-711, 2008.

SUMMARY: Overweight and obesity in Chile have become an epidemic which exceeds all social sectors. The main factors have been the change in lifestyle, economy, sedentary and genetic. The purpose of this study was analysing the BMI and Somatotype in a sample of overweight and obese adolescents in Temuco city. 50 adolescents were assessed, both sexs (19 men and 31 women) between 14 and 16 years old belonging to subsidized schools of Temuco city, IX Region of Chile. The adolescents were evaluated on their schools. To obtain the somatotype the anthropometric of Heath \& Carter method was used, classifying BMI according to the WHO norms. Women were more endomorphofic than men, this difference was statistically significant. The mesomorphic and ectomorphic component didn't show statistically significant differences between sexes. The BMI value showed an average of 27.8; the minimum value was 23 and the maximum value was 35 , the mode was 30.6. The sample presented a prevalence of the endomorphofic component. These results show the existence of a great amount of adipose tissue within adolescents. Then, they were more overweight and obese. These factors are due to the lack of physical activity, change in food habit, and different other reasons; such as, obese parents, genetics, etc.

KEY WORDS: BMI; Somatotype.

\section{REFERENCIAS BIBLIOGRÁFICAS}

Almagià, F. A.; Gurovich, M. A.; Ivanovic, M. D.; Toro, D. T. \& Binvignat, G. O. Estudio y análisis morfológico y etario del dimorfismo sexual a través de la composición corporal. Rev. Chil. Anat., 15(2):141-9, 1997.

Almagià, F. A; Toro, D. T.; Binvignat, G. O.; Cabrera, E. \& Marinao, A. Aproximación al perfil morfoestructural y dismorfismo sexual de jóvenes de ambos sexos de edad de 6 a 23 años de edad caracterizados por el somatotipo. Rev. Chil. Anat., 14(2):189-97, 1996.

Andersen, E. The spread of the childhood obesity epidemic. JAMC., 63:1461-2, 2000.

Burrows; R. Díaz, N.; \& Muzzo, S. Variaciones del índice de masa corporal (IMC) de acuerdo al grado de desarrollo puberal alcanzado. Rev. méd. Chile, 132(11): 13638, 2004.

Burrows, R.; Burgueno, M.; Leiva, L.; Ceballos, I.; Gattas, V.; Lera, L. \& Albala, C. Perfil metabólico de riesgo cardiovascular en niños y adolescentes obesos con menor sensibilidad insulínica. Rev. méd. Chile, 133 (7),:795$804,2005$.

Caitlin, B. \& Fergus, C. Critical reviews in food science and nutrition. 45:511-25, 2005.

Depress, P. Visceral obesity, insulin resistance and dyslipidemia: contribution of endurance exercise training to the treatment of the plurimetabolic syndrome. Exercise and Sport Scienc., 25:271-300, 1997.

Ebbeling B.; Pawlak, B. \& Ludwig, S. Childhood obesity: Public-health crisis, common sense cure. Lancet, 360:473-82, 2002.

Flegal, K. M.; Carroll, M. D.; Ogden, C. L. \& Johnson, C. L. Prevalence and trends in obesity among US adults, 999-2000. JAMA, 288(14):1723-7, 2002.

Katzmarzyk, P. \& Malina, R. Body size and physique among 
Canadians of first nation and Europeans ancestry. Am. J. Phys Anthrpol., 108:161-72, 1999.

Katzmarzyk, P.; Malina, R.; Song, T.; Theriault, G. \& Bouchard, C. Physique and echocardiography dimensions in children, adolescent and young adults. Ann. Hum. Biol., 25:145-57, 1998.

Global prevalence and secular trends in obesity. In: Obesity. Preventing and managing the global epidemic. Report of a WHO consultation on obesity. Geneva 3-5 June, 17-36. 1997.

Magnusson, C.; Baron, J.; Persson, I.; Wolf, A. Bergstrom, R.; Trichopoulos, D. \& Adami, H. Body size in different periods of life and breast cancer risk in post-menopausal women. Int. J. Cáncer, 76:29-34, 1998.

Muzzo, S.; Cordero, J.; Ramírez, I. \& Burrows R. Tendencia secular del estado nutricional en escolares chilenos. Rev. Chil. Nutr., 26:311-5. 1999.

Silva, H.; Bruneau, J. C.; Reyno, H. P. \& Bucarey, S. Somatotipo e índice de masa corporal en una muestra de adolescentes de ambos sexos de la ciudad de Temuco, Chile. Int. J. Morphol., 21(4):309-13, 2003.

Swang, P. \& McConnell, K. Anthropometry and bioelectrical impedance inconsistently predicts fatness in women with regional adiposity. Med. Scienc. Sport. Exerc., 31(7):1068-75, 1999.

Toro, T. \& Almagià, A. Aplicación de estándares antropométricos para la evaluación del crecimiento y estado nutricional en niñas y niños de 11-15 años de Valparaíso. An. Anat. Normal, 7:99-107, 1989.

Toro, T.; Arenas G. \& Almagià, A. Caracterización somatotípica de jóvenes estudiantes de Valparaíso. An. Anat. Normal, 1:101-6, 1983.
Veugelers, P. \& Fitzgerald, A. Prevalence of risk factors for childhood overweight and obesity. JAMC, 173(6):60713,2005 .

Wang, J.; Thornton, J. \& Burastero, M. Asians have lower body mass index (BMI) but higher percent body fat than do whites: Comparisons of anthropometric measurements. Am. J. Clin. Nutrition, 43(1):23-8, 1994.

White, E.; Wilson, A.; Maccopwan, G.; Thomas, G.; Cairns, A.; \& Rickets, I. Body mass index centiles charts to assess fatness of British children. Arch. Dis. Child., 72: 38-41, 1995.

WHO Technical report series 916. Diet, nutrition and the prevention of chronic diseases. Report of a joint FAO/ WHO Expert Consultation. World Health Organization, Geneva, 2003.

\section{Dirección para Correspondencia: \\ Prof. Héctor Silva Mella \\ Facultad de Medicina \\ Universidad de La Frontera \\ Casilla 54-D \\ Temuco - CHILE \\ Email:hsilva@ufro.cl}

Recibido : 25-04-2008

Aceptado: 14-07-2008 